\title{
Simulation and Experiments to Identify Factors Allowing Synthetic Control of Structural Features of Polymeric Nanoparticles Supplementary Information
}

\author{
William C. Swope, Julia E. Rice, Victoria A. Piunova, \\ Amber C. Carr, Robert D. Miller, Joseph Sly \\ IBM Almaden Research Center \\ 650 Harry Road, San Jose, CA 95120
}

June 10, 2016

\section{Force Field and Model Details}

The development of coarse grained structural models and force field parameters for the reactants was actually quite challenging. As much as possible we wanted coarse graining at an OPLS united atom (OPLS-UA) level of granularity. The arms were modeled with structures and parameters based on OPLS-UA representations of alkanes and alcohols. However, initial attempts to model the linker molecular components with OPLS-UA were not successful when the structural model was based on the reactant double closed ring structure, where the carbonyl carbon and the oxygen of the ester group remained close during the simulation. This is because each reaction with a closed ring of the linker actually causes it to open, after which the resultant hydroxyl moiety can be quite far from the ester bond that just formed and caused the ring opening. That hydroxyl group needs to be far enough away from the ester group to avoid being crowded and allow it to be involved in subsequent reactions. Therefore, we built the structural model for the linker 
based on all atom models of ring-opened forms of the linker. These opened structures provided coordinates for the reacting sites (the carbonyl and oxygen groups), and a six site framework was mapped to this for the coarse grained model of the linker. This framework determined the bond lengths used in the six site model, but angle bend and torsion motion was allowed through soft intramolecular parameters, allowing considerable flexibility. At the start of simulations, the carbonyl site of the ester is reactive, but the oxygen site is not. Then, after the carbonyl site reacts, the adjacent oxygen site is modified so that it can later to react. The carbonyl and oxygen sites on the linker framework were given LJ parameters consistent with OPLS-AA.

Solvent was not included in the simulation, so, in order to mimic the dielectric shielding effect of solvent, all charges on arm and linker sites were set to zero. Finally, to model a good solvent, all the LJ interactions were truncated and shifted to be continuous at the truncation distance, except for pairs of sites that are capable of reacting, where a small attractive interaction was allowed. This resulting model captures in a general way the volume, topology and flexibility of the reactant molecules. These coarse grained models have sufficient structural detail to allow an all atom structural model to be mapped onto them for subsequent simulations with more accurate force fields.

\section{$1.1 \quad$ Implicit Solvent}

Solvent molecules were not explicitly included in the simulations, but their effect was introduced into the interactions between reactant molecules through the use of modified Lennard-Jones functional forms that were truncated at the Lennard-Jones minimum and shifted to be continuous at the truncation distance, in the spirit of a WCA potential [John D. Weeks, David Chandler and Hans C. Andersen Role of Repulsive Force in Determining the Equilibrium Structure of Simple Liquids", Journal of Chemical Physics 54 5237-5247 (1971)]. This has the effect of approximating a noninteracting but good solvent, of the sort played by dichloromethane in the experiment. Interactions between reacting sites (such as between those representing hydroxyl groups and carbonyl groups) were adjusted from this general approach by making them slightly attractive and allowing a longer range for their interaction 


\subsection{Arms}

Arms were models of aliphatic alcohols, $\mathrm{H}-\left(\mathrm{CH}_{2}\right)_{n}-\mathrm{OH}$ with $\mathrm{n}=8$ (short chains, octanol) or $\mathrm{n}=16$ (longer chains, hexadecanol). Coarse grained models of arms consisted of three types of sites, designated C1 (terminal united atom methyl), C2 (united atom methlyene), and $\mathrm{O}$ (united atom hydroxyl group). For these the mass (amu), and Lennard Jones parameters $(\sigma, \AA ; \epsilon, \mathrm{kcal} / \mathrm{mole})$

\begin{tabular}{|lccc|} 
are \\
\hline Type & Mass & $\sigma$ & $\epsilon$ \\
\hline C1 & 14 & 3.905 & 0.118 \\
C2 & 14 & 3.905 & 0.118 \\
O & 17 & 3.070 & 0.170 \\
\hline
\end{tabular}

Unless noted, geometric combining rules were used: $\sigma_{i j}=\sqrt{\sigma_{i i} \sigma_{j j}}$ and $\epsilon_{i j}=\sqrt{\epsilon_{i i} \epsilon_{j j}}$. All LJ interactions were truncated at the minimum of the LJ potential $\left(\mathrm{R}_{c}=2^{1 / 6} \sigma\right)$, and shifted to provide a smooth and continuous repulsive interaction. All charges are zero.

Bond energies $\left(U(\mathrm{R})=0.5 k\left(\mathrm{R}-\mathrm{R}_{e}\right)^{2}\right)$ with spring constants $\left(\mathrm{k},(\mathrm{kcal} / \mathrm{mole})-\AA^{-2}\right)$ and equilibrium lengths $\left(\mathrm{R}_{e}, \AA\right)$ were computed using the following parameters:

\begin{tabular}{|lcc|}
\hline & $\mathrm{k}$ & $\mathrm{R}_{e}$ \\
\hline $\mathrm{C} 1-\mathrm{C} 2$ & 260 & 1.526 \\
$\mathrm{C} 2-\mathrm{O}$ & 386 & 1.425 \\
\hline
\end{tabular}

Angle energies $\left(U(\theta)=0.5 k\left(\theta-\theta_{0}\right)^{2}\right)$ with spring constants $\left(\mathrm{k},(\mathrm{kcal} / \mathrm{mole})-(\mathrm{Rad})^{-2}\right)$ and equilibrium angles $\left(\mathrm{R}_{e}\right.$, degrees) were computed using the following parameters:

\begin{tabular}{|lcc|}
\hline & $\mathrm{k}$ & $\theta_{0}$ \\
\hline $\mathrm{C} 1-\mathrm{C} 2-\mathrm{C} 2$ & 63 & 112.4 \\
$\mathrm{C} 2-\mathrm{C} 2-\mathrm{C} 2$ & 63 & 112.4 \\
$\mathrm{C} 2-\mathrm{C} 2-\mathrm{O}$ & 80 & 109.5 \\
\hline
\end{tabular}

Torsion energies $\left(U(\phi)=c_{1}(1+\cos (\phi))+c_{2}(1-\cos (2 \phi))+c_{3}(1+\cos (3 \phi))\right)$ with coefficients $c_{i}$ (kcal/mole) were computed using the following parameters:

\begin{tabular}{|lccc|}
\hline & $c_{1}$ & $c_{2}$ & $c_{3}$ \\
\hline C1-C2-C2-C2 & 0.70550 & -0.13550 & 1.5725 \\
C2-C2-C2-O & 0.35150 & -0.10600 & 1.5300 \\
\hline
\end{tabular}

Most parameters are from OPLS-UA (united atom) JACS 106, 6638 (1984). 


\subsection{Linker}

In this six site model, sites 1,2 , and 3 are at one end of the linker molecule, and sites 4, 5, and 6 are at the other end. Sites 1 and 5 start out with site types $\mathrm{A}$ and $\mathrm{E}$ and represent carbonyl carbon sites located at the end of $\left(-\mathrm{CH}_{2}-\mathrm{CH}_{2}-(\mathrm{CO})-\right)$ groups. Sites 2 and 6 start out with site types B and $\mathrm{F}$ and represent the oxygen sites in the ring connected to $\left(-\mathrm{CH}_{2}-\mathrm{O}-\right)$ groups. Finally, sites 3 and 4 are of types $\mathrm{C}$ and $\mathrm{D}$ and represent the (-CH-) groups at the ends of the single bond that joins the two rings.

\begin{tabular}{|ccccc|}
\hline Site & Type & Mass & $\sigma$ & $\epsilon$ \\
\hline 1 & A & 56 & 3.75 & 0.105 \\
2 & B & 30 & 3.00 & 0.170 \\
3 & C & 13 & 3.50 & 0.066 \\
4 & D & 13 & 3.50 & 0.066 \\
5 & E & 56 & 3.75 & 0.105 \\
6 & F & 30 & 3.00 & 0.170 \\
\hline
\end{tabular}

These are the Lennard-Jones OPLS-AA parameters for ester sites (J. Comp. Chem. 22, 1340, 2001). Ester carbonyl carbon sites 1 and 5 of types A and $\mathrm{E}$ are known to OPLS-AA as type "C", the ring ester oxygen sites 2 and 6 of types B and F are known to OPLS-AA as type "OS", and the linker carbon sites 3 and 4 of types $\mathrm{C}$ and D are known to OPLS-AA as type "CT".

Bond energies were computed using the following parameters:

\begin{tabular}{|ccc|}
\hline & $\mathrm{k}$ & $\mathrm{R}_{e}$ \\
\hline $1-3$ & 268 & 3.500 \\
$2-3$ & 268 & 2.300 \\
$3-4$ & 268 & 1.526 \\
$4-5$ & 268 & 3.500 \\
$4-6$ & 268 & 2.300 \\
\hline
\end{tabular}

Angle energies were computed using the following parameters:

\begin{tabular}{|ccc|}
\hline & $\mathrm{k}$ & $\theta_{e}$ \\
\hline $1-3-2$ & 63 & 120 \\
$1-3-4$ & 63 & 120 \\
$2-3-4$ & 63 & 120 \\
$5-4-6$ & 63 & 120 \\
$3-4-5$ & 63 & 120 \\
$3-4-6$ & 63 & 120 \\
\hline
\end{tabular}

This model has two improper torsion terms $U(\phi)=10.5(1+\cos (\phi))$ 
(kcal/mole), for torsion angles 1-2-3-4 and 3-4-6-5 that serve to keep each of these two groups of four sites relatively flat. There are no torsion terms restricting rotation about the $3-4$ bond.

\subsection{Interactions Formed And Modified During Chem- ical Reactions}

At regular time intervals (every 1000 molecular dynamics time steps) during the simulation, distances between all pairs of potentially reacting sites were measured and when two such sites were sufficiently close (less than $2.6 \AA$ ), a bond was added between them through the addition of a new force field term. Reacting sites on the coarse grained molecules represented either hydroxyl groups (alcohol groups at the ends of arms or on ring opened lactone linkers) or carbonyl groups in closed rings of the lactone linker. Each of these sites could be in one of several different reactive states. For example, sites on unreacted arms were capable of reacting with unreacted carbonyl sites on the linkers, but immediately after such a reaction occurred, both of the sites changed to an unreactive state to prevent them from further reacting with other material in the system. All changes of a sites state were accompanied by changes in the Lennard-Jones parameters used for their interactions.

After every 100 cycles of these kinds of bond forming reactions (i.e., after 100000 steps of the simulation) a different type of state change was enabled for some of the other sites. After a carbonyl site on a lactone had reacted with a hydroxyl group at some time during the previous 100000 steps of the simulation, the state of the adjacent site on the lactone, representing an oxygen atom, was changed from unreactive to reactive, now allowing it to potentially react with other unreacted carbonyl sites on the linkers in the system. Over the course of the simulation, therefore, sites on the coarse grained model of the linker representing ring oxygen atoms changed state from unreactive to reactive representing that a reaction had occurred, and then back again to unreactive after another reaction has occurred. After these oxygen sites were activated, the whole process was repeated (the simulation was continued for another 100 cycles of bond forming reactions, performed every 1000 molecular dynamics steps). Simulations were stopped after reactions were no longer observed, usually after every lactone ring was bonded to at least one other site. 
This process was implemented as follows: When a reactive arm alcohol oxygen site (site type $\mathrm{O}$ ) is within $2.6 \AA$ of a reactive carbonyl (sites 1 or 5 , having site types $\mathrm{A}$ or $\mathrm{E})$, a bond is formed ( $\mathrm{k}=100$ (kcal $/ \mathrm{mole})-\AA^{-2}$; $\mathrm{R}_{e}=2.5 \AA$ ) between them, and the alcohol oxygen site type is changed from $\mathrm{O}$ (reactive) to $\mathrm{Z}$ (making it inactive), and the carbonyl site (either 1 or 5 ) is changed from type $\mathrm{A}$ or $\mathrm{E}$ (reactive) to type $\mathrm{G}$ (inactive).

Periodically, a force field editing step is performed that changes a ring oxygen on site 2 or 6 from an inactive state (type $\mathrm{B}$ or $\mathrm{F}$ ) to a reactive hydroxyl state (type $\mathrm{H}$ ). This is done only if the adjacent carbonyl (site 1 or 5) has previously changed from reactive (type A or E) to inactive (type G) as a result of having formed a bond to either an alcohol arm or to another lactone.

Reactive carbonyl groups (sites 1 or 5 , if they are of type A or E) can also form bonds with reactive hydroxyl groups (sites 2 or 6 , when they are of type $\mathrm{H}$ ). When a reactive carbonyl site (with type $\mathrm{A}$ or $\mathrm{E}$ ) is within $2.6 \AA$ of a reactive hydroxyl site (with type $\mathrm{H})$, a bond is formed $\left(\mathrm{k}=100\right.$ (kcal/mole)- $\AA^{2}$ ; $\mathrm{R}_{e}=2.5 \AA$ ) between them, and the carbonyl site is changed from type A or $\mathrm{E}$ (reactive) to type $\mathrm{G}$ (inactive), and the hydroxyl site is changed from type $\mathrm{H}$ (reactive) to type I (inactive).

Parameters for new site types that arise from reactions:

\begin{tabular}{|cccccl|}
\hline Site & Type & Mass & $\sigma$ & $\epsilon$ & Description \\
\hline O & Z & 17 & 2.00 & 0.170 & inactivated alcohol oxygen \\
2,6 & $\mathrm{H}$ & 30 & 2.00 & 0.170 & activated hydroxyl oxygen \\
1,5 & $\mathrm{G}$ & 56 & 3.00 & 0.105 & inactivated carbonyl carbon \\
2,6 & $\mathrm{I}$ & 30 & 2.00 & 0.170 & inactivated hydroxyl oxygen \\
\hline
\end{tabular}

Unless previously noted, all LJ interactions use the same geometric combining rules, and they are truncated at the minimum and shifted to produce a short ranged smooth and continuous potential, except for the following:

\begin{tabular}{|ccccc|}
\hline$i$ & $j$ & $\sigma$ & $\epsilon$ & $\mathrm{R}_{c}$ \\
\hline $\mathrm{A}$ & $\mathrm{O}$ & 2.44948974 & 0.170 & 9.00 \\
$\mathrm{E}$ & $\mathrm{O}$ & 2.44948974 & 0.170 & 9.00 \\
$\mathrm{~A}$ & $\mathrm{H}$ & 2.44948974 & 0.170 & 9.00 \\
$\mathrm{E}$ & $\mathrm{H}$ & 2.44948974 & 0.170 & 9.00 \\
\hline
\end{tabular}

These interactions have been modified to be longer ranged and more attractive in order to promote reactions between sites of these types. They are truncated at $\mathrm{R}_{c}$ and shifted to produce a smooth and continuous potential.

Parameters for bonds that arise from reactions: 


\begin{tabular}{|cccl|}
\hline & $\mathrm{k}$ & $\mathrm{R}_{e}$ & Description \\
\hline O-(1,5) & 100 & 2.50 & arm-linker bond \\
$(2,6)-(1-5)$ & 100 & 2.50 & linker-linker bond \\
\hline
\end{tabular}

\section{Simulation Parameters}

Molecular dynamics simulations were performed with a version of the LAMMPS simulation package [Plimpton, S. (1995) Fast Parallel Algorithms For ShortRange Molecular-Dynamics, J. Comp. Phys. 117, 1-19.] dated July 2009, and extensive use was made of the bond/create feature of this version of the software, which supports the bond formation between specific reactive sites that was performed every 1000 molecular dynamics time steps. The editing of atomic site types based on bonding patterns that was performed every 100000 steps was done using software developed in house. The LAMMPS software has been efficiently parallelized for the IBM BlueGene/L supercomputer used for the simulations. All simulations were performed under the NVT ensemble, using a control temperature of 400K and the Nose-Hoover extended Lagrangian thermal control algorithm, with a fictitious mass set so as to establish a fluctuation period [Martyna, G. J., Tobias, D. J., And Klein, M. L. (1994) Constant-Pressure Molecular-Dynamics Algorithms, J. Chem. Phys. 101, 4177-4189.] of approximately $100 \mathrm{fs}$ in the thermostat variable (known as the thermostat damping factor in LAMMPS). The dynamical integration scheme was velocity-Verlet [Swope, W. C., Andersen, H. C., Berens, P. H., And Wilson, K. R. (1982) A Computer-Simulation Method For the Calculation of Equilibrium-Constants For the Formation of Physical Clusters of Molecules - Application To Small Water Clusters, J. Chem. Phys. 76, 637-649.] with a time step of $1 \mathrm{fs}$. The analysis of the resulting trajectory data was performed on a local IBM Power workstation cluster.

The starting configurations for each simulation were produced by random placement of the coarse grained arm and linker molecules on sites of a simple cubic lattice in numbers to give the desired reactant ratios and densities. To equilibrate, simulations of 1 ns (a million molecular dynamics steps) were performed on these starting states for mixing and thermal equilibration using a force field modified to have all intermolecular attraction turned off. The intermolecular attractions were then turned on and bonds were allowed to form. During the bond formation stages of the simulations, the growing list of bonds was written to disk every 1000 time steps and the coordinates every 10000 time steps for subsequent analysis. This allowed for the identification 
of individual growing polymer molecules with time, the time of formation and nature of each bond, and detailed structural characterizations of each polymer molecule in the ensemble that formed. These included size, arm/linker composition, shape metrics, including the radius of gyration, and the number and types of bonds of various types. Distributions of these metrics were produced over the course of the synthetic process and characterized at each time by number and weight averaged mean sizes and polydispersity indices.

\section{Universal Scaling From First Order Kinet- ics}

Here we derive some kinetic relationships that lead to simplifications in the analysis. This analysis addresses the case where some number of arm and cross linker molecules are mixed together in a solvent, along with sufficient catalyst. In our case, the arms are chain molecules with an alcohol end group, and the linker is a bis-lactone molecule, but other scenarios are similarly described. The way these chain reactions proceed, each reaction consumes a hydroxyl group and produces another one somewhere else. The number of hydroxyl groups, therefore, is conserved and is equal to the number of arm molecules present at the start of the reaction. We denote the concentration of hydroxyl groups in the reaction mixture as $[\mathrm{ROH}]$. Similarly, we represent the concentration of linker reactive sites present at time $t$ as $[\mathrm{X}]_{t}$. At the start of the reaction, the number of cross linker reactive sites, $[\mathrm{X}]_{0}$ is just twice the number of linker molecules, but these get consumed as the reaction proceeds.

Simple first order kinetics is obtained since the rate of consumption of linker reactive sites will be linear in the concentration of these sites, as well as linear in (constant) concentration of hydroxyl groups:

$$
\frac{d[\mathrm{X}]_{t}}{d t}=-k_{r}[\mathrm{ROH}][\mathrm{X}]_{t}
$$

where $k_{r}$ is the rate constant. The solution of this equation is the following:

$$
[\mathrm{X}]_{t}=[\mathrm{X}]_{0} \exp \left(-k_{r}[\mathrm{ROH}] t\right)
$$

The number of bonds formed after time $t$ is equal to the number of linker reactive sites consumed, $\left([\mathrm{X}]_{0}-[\mathrm{X}]_{t}\right) V=[\mathrm{X}]_{0} V\left\{1-\exp \left(-k_{r}[\mathrm{ROH}] t\right)\right\}$. Where 
$V$ is the volume of solution in the reaction vessel. So, the initial concentration of cross linker determines the amplitude of the behavior, and the initial concentration of arms determines the speed of the behavior. In comparing situations with variations in the overall reactant concentration and/or in the ratio of reactants, some of the differences are due simply to these concentration effects. To compare situations with different cross linker concentration, it will be convenient to plot $\left(1-[\mathrm{X}]_{t} /[\mathrm{X}]_{0}\right)$. To compare situations with different arm concentrations, is will be convenient to plot observables as a function of a scaled time, $t^{\prime}=\left([\mathrm{ROH}] /[\mathrm{ROH}]_{\text {ref }}\right) t$, where $[\mathrm{ROH}]_{\text {ref }}$ is some convenient concentration common to all cases being compared. With this substitution, the fraction of linker sites consumed, the extent of the reaction, is given by the following:

$$
\frac{[\mathrm{X}]_{0}-[\mathrm{X}]_{t}}{[\mathrm{X}]_{0}}=e^{-k_{r}^{\prime} t^{\prime}}
$$

where $k_{r}^{\prime}=k_{r}[\mathrm{ROH}]_{\text {ref }}$ is a modified rate constant. Using this approach, observables for many different reactant concentrations and reactant ratios can fit on a common universal curve.

\section{Number and Size-weighted Averages}

The number weighted and size weighted polymer sizes, as well as the polydispersity index, were computed as follows.

Polymers were defined as molecules with at least one arm and one linker component. (Otherwise, the molecule was considered to be either an unreacted arm or linker.) Given $N$ such polymer molecules, each with $a_{i}$ arm and $l_{i}$ linker components, where $a_{i} \geq 1$ and $l_{i} \geq 1(i=1, \ldots, N)$, the number weighted average size, $S_{n}$, and size weighted average size, $S_{w}$, are given by the following:

$$
\begin{aligned}
S_{n} & =\frac{1}{N} \sum_{i=1}^{N}\left(a_{i}+l_{i}\right) \\
S_{w} & =\frac{\sum_{i=1}^{N}\left(a_{i}+l_{i}\right)^{2}}{\sum_{i=1}^{N}\left(a_{i}+l_{i}\right)}
\end{aligned}
$$

The size polydispersity index (PDI) was computed from these as $P_{s}=S_{w} / S_{n}$. These are the results generally reported in this paper. 
Since the number of linker components per arm component is a sharply peaked function related to their ratio in the initial reactant mixture, we can approximate $l_{i} \approx \alpha a_{i}$, where $\alpha$ represents that ratio. Using this amounts to neglecting contributions to the dispersity from variation in the linker to arm ratio within the polymers, and considering only that due to variation in the number of arms. With this approximation, one obtains the following:

$$
\begin{aligned}
& S_{n} \approx(1+\alpha) \frac{1}{N} \sum_{i=1}^{N} a_{i} \\
& S_{w} \approx(1+\alpha) \frac{\sum_{i=1}^{N} a_{i}^{2}}{\sum_{i=1}^{N} a_{i}}
\end{aligned}
$$

The size polydispersity index, $S_{w} / S_{n}$, is therefore seen to be equivalent to the polydispersity in the arm count.

These polymer size metrics can be related to the standard number, $M_{n}$, and mass weighted, $M_{w}$, molecular weight of the polymer mixture. If the molecular weight of an arm and linker component is $m_{a}$ and $m_{l}$, respectively, the average polymer molecular weights are defined as follows:

$$
\begin{aligned}
& M_{n}=\frac{1}{N} \sum_{i=1}^{N}\left(m_{a} a_{i}+m_{l} l_{i}\right) \\
& M_{w}=\frac{\sum_{i=1}^{N}\left(m_{a} a_{i}+m_{l} l_{i}\right)^{2}}{\sum_{i=1}^{N}\left(m_{a} a_{i}+m_{l} l_{i}\right)}
\end{aligned}
$$

The polydispersity index is usually computed as the ratio of these molecular weight averages, $P_{m}=M_{w} / M_{n}$. However, using the approximation that the linker content is proportional to the arm content, these relationships simplify to the following:

$$
\begin{aligned}
& M_{n} \approx\left(m_{a}+\alpha m_{l}\right) \frac{1}{N} \sum_{i=1}^{N} a_{i} \\
& M_{w} \approx\left(m_{a}+\alpha m_{l}\right) \frac{\sum_{i=1}^{N} a_{i}^{2}}{\sum_{i=1}^{N} a_{i}}
\end{aligned}
$$

So, under the assumption of linker to arm proportionality, one can see that the familiar polydispersity computed from the molecular weight ratios, $M_{w} / M_{n}$, is equivalent to that computed from the size ratios, $S_{w} / S_{n}$, and reflects the variation in the arm content. Moreover, this metric is insensitive to the masses of the arms and linker. 


\section{Supplementary Figures and Diagrams}

Figure S1 shows example star polymer structures from short arm simulations with intermediate reactant density and an arm to linker reactant ratio of 1:5. The smaller polymer in the figure has 17 arms, 77 linkers; the larger one has 35 arms, 177 linkers. Hydroxyl groups are indicated with orange spheres. The coloring indicates qualitatively when the various components joined this polymer; blue regions formed early, then white, and finally red.

Figure S2 shows example star polymer structures from the long arm simulations with intermediate reactant concentration and arm to linker reactant ratio of 1:5. This figure is an alternate view of the same two polymers seen in Figure 17 of the manuscript, but drawn here to the same scale. In this figure, the arm material is colored blue and the linker material is colored red. Figure S3 Example star polymers from the end of simulations with intermediate reactant density and, from top to bottom, arm to linker ratios of 1:1 (21 arm, 30 linker components), 1:2 (36 arm, 89 linker) and 1:7 (35 arm, 232 linker).

Figure S4 shows an example of ladders, common structural motifs seen in star polymers made by the simulations. On the left is a more complete chemical representation of a growing ladder; on the right is a coarse grained representation seen in the simulations. The structure results when a linker bonds twice to a linker previous in a chain. The structure terminates with two hydroxyl groups at the end of a growing ladder. Ladders projecting from a linker-rich core region into the solvent can readily extend by addition of more linker from solvent, and they may play a role in the merging process when two star polymers combine to make a larger one. Arms are not shown on the coarse grain model for clarity.

Figure S5 shows the numbers of bonds formed of type 1 (upper panel), between unreacted arms and unreacted linker, and of type 2 (lower panel), between unreacted arms and linker molecules in growing polymers, as a function of scaled time during simulations at intermediate total reactant density for a range of arm to linker reactant ratios. The arm to linker reactant ratios on the upper panel are, from bottom to top $7: 1,7: 2,1: 1,1: 2,1: 3,1: 5$, and 1:7. In the lower panel, they are, from bottom to top at $6 \mathrm{~ns} 1: 7,1: 5,7: 1$, and 1:3. Times for each curve in these graphs were scaled differently, by the arm concentration for that composition, $[\operatorname{Arm}(1: \mathrm{x})] /[\operatorname{Arm}(1: 5)]$ where $[\operatorname{Arm}(1: \mathrm{x})]$ is the initial arm concentration for simulations with an arm to linker concentration ratio of 1:x. Each simulation had 7000 arm molecules and a different 


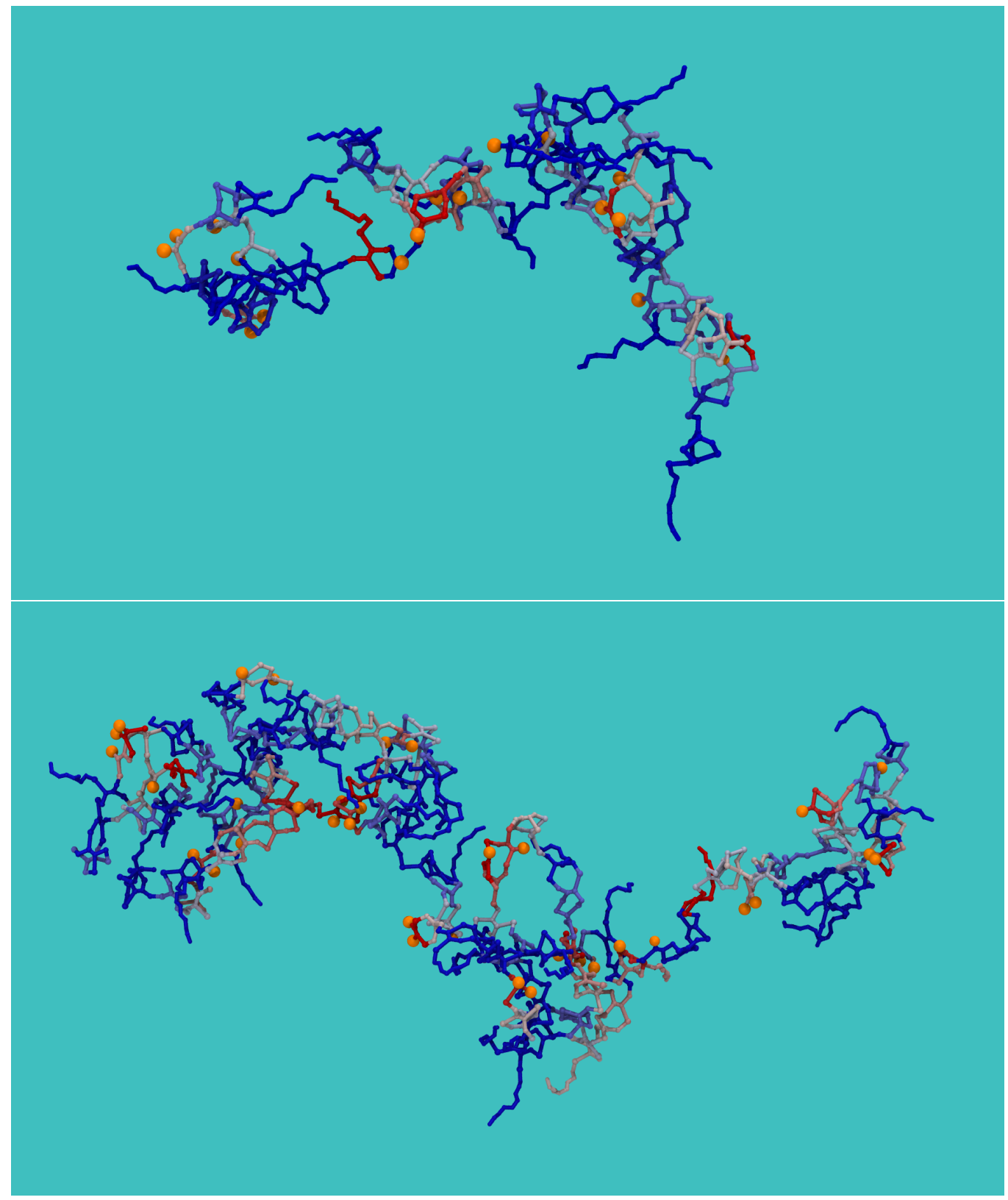

Figure S1: Example star polymer structures from short arm simulations with intermediate reactant density and an arm to linker reactant ratio of 1:5. 


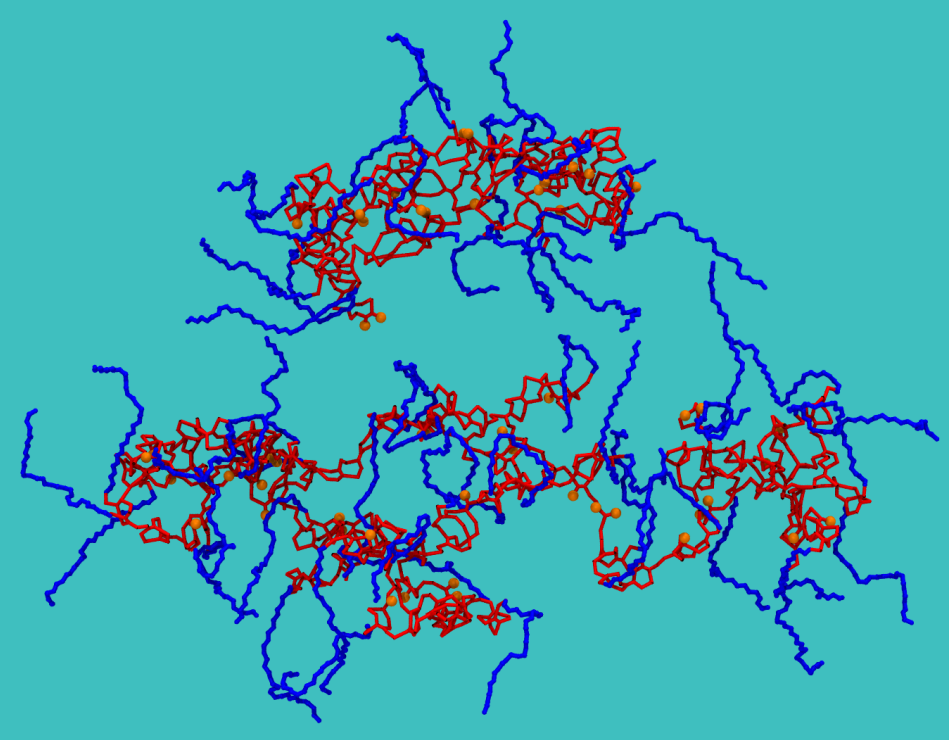

Figure S2: Example star polymer structures from long arm simulations with intermediate reactant density and an arm to linker reactant ratio of 1:5.

amount of linker to give the desired reactant ratio.

The sum of the numbers of these two bond types in a simulation gives the number of arm molecules that have reacted since bonds involving arms are either of type 1 (formed between previously unreacted arm and unreacted linker molecules) or type 2 (addition of an unreacted arm to a linker on a growing polymer), the sum of the numbers of these two bond types in a simulation gives the number of arm molecules that have reacted. Analysis of the formation of these bonds shows that in most cases, reactions with arms have nearly stopped by about 2 ns into these simulations. Since there are 7000 arm molecules in each of these simulations, the numbers of bonds of these types can be compared directly. In the most arm rich environment (7:1), there are 7000 arm molecules and only 1000 linker molecules. Figure S5 shows that 909 bonds of type 1 form, nearly one arm per linker, when each of 909 linkers gets a first arm. Figure S5 shows that 697 bonds of type 2 form in this simulation, most resulting from the addition of a second arm to many of these linker molecules, producing a large number of small polymers with a single linker and two arms. (These numbers also suggest that only 


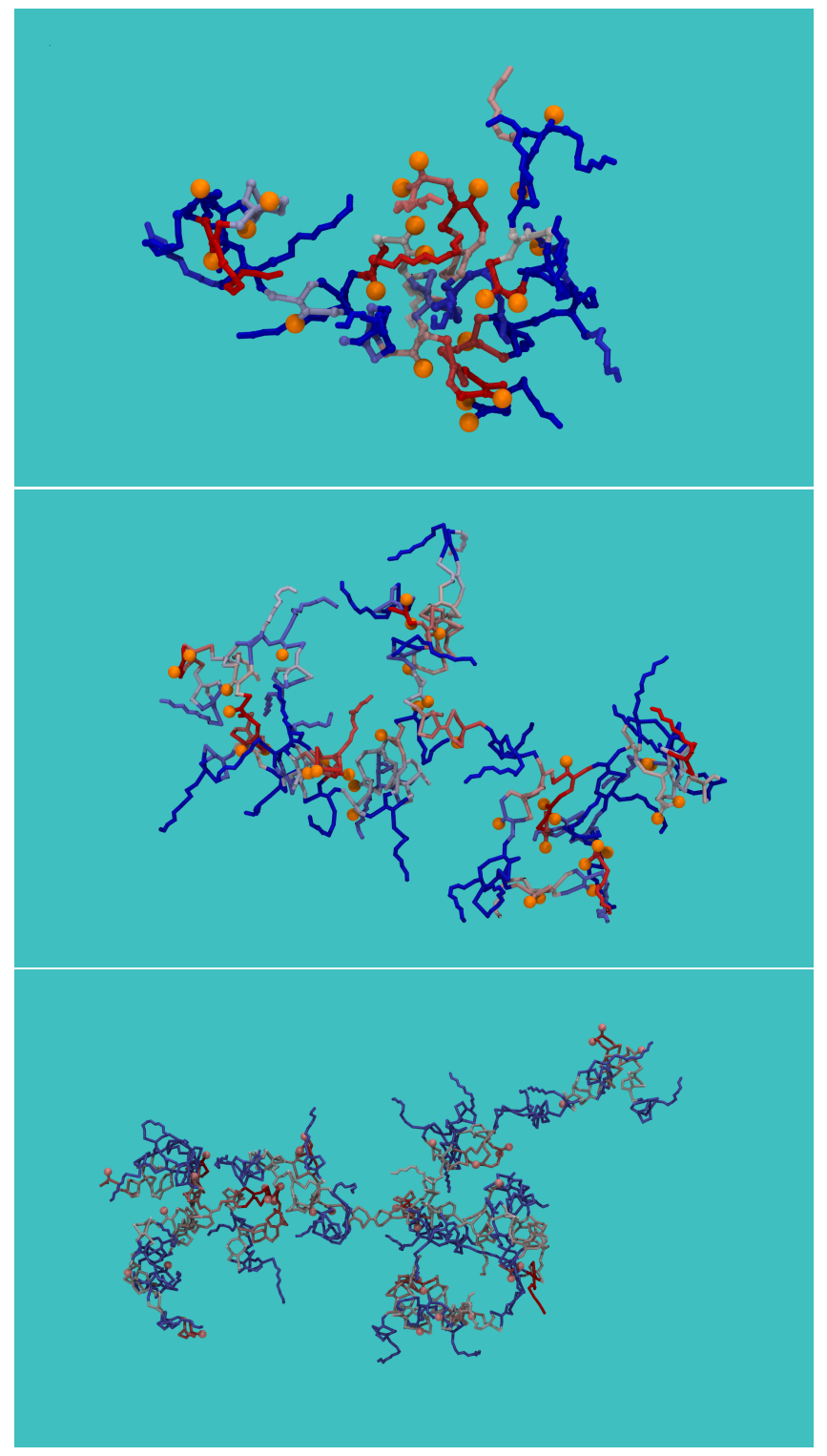

Figure S3: Example star polymer structures from short arm simulations with intermediate reactant density and various arm to linker reactant ratios of $1: 1$, $1: 2$ and $1: 7$.

1606 of the 7000 arms react, out of a maximum possible of 2000, since there are 1000 linker molecules.) These polymer constructs cannot undergo further 


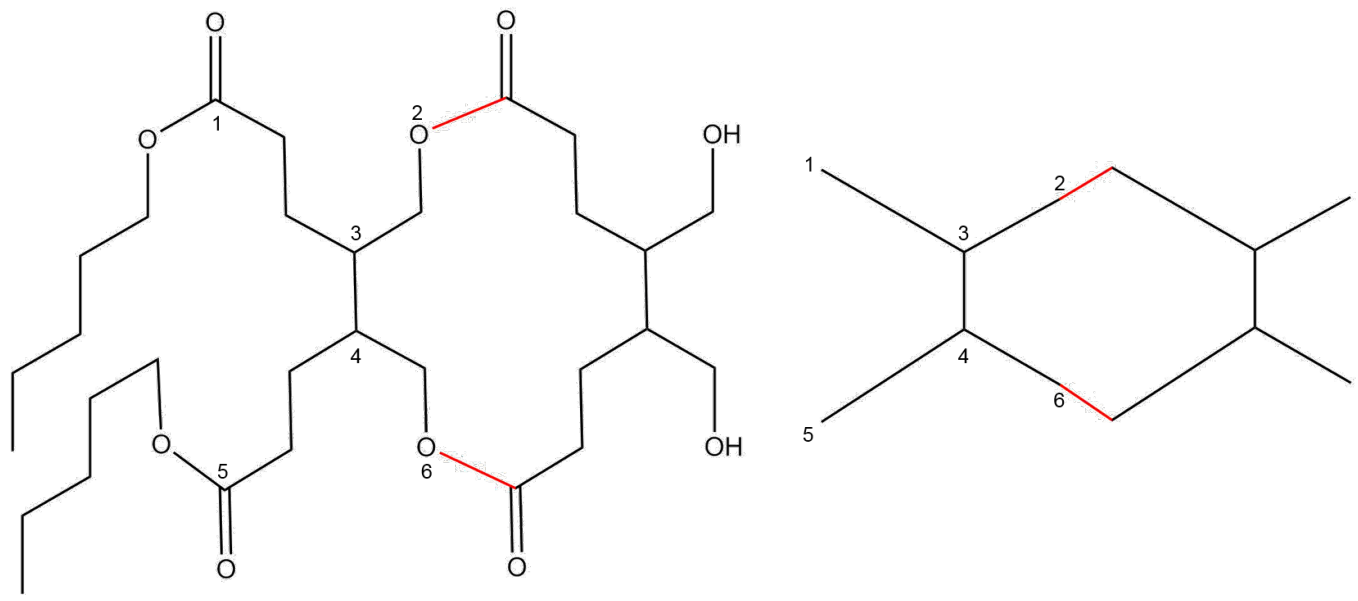

Figure S4: Ladders are common structural motifs seen in star polymers made during simulations.

growth in an arm rich environment. Since each linker molecule is capable of bonding no more than two arms, this linker-limited situation also occurs in the 7:2 simulation (with 7000 arms and 2000 linkers), where 1634 bonds of type 1 and 1805 of type 2 form. These two most arm rich systems do not have enough linker material to produce very large polymers, such that the largest polymers produced in these situations consist of only a few tens of molecular components. Note, however, that these polymers will have an arm to linker ratio close to 2:1 (two arms per linker), regardless of the exact arm and linker composition.

In contrast, the other five compositions are not linker limited in this way. In these simulations, starting with the $1: 1$ ratio (7000 arms and 7000 linkers) and proceeding to higher relative linker content, after most of the arms have been consumed, one finds in addition to linkers having two arms, there will also be unreacted linker material as well as some unreacted sites on linkers that already have a single arm. The relative amounts of unreacted linker, nascent polymers consisting of a linker with a single arm component, and those with two arms will depend sensitively on the initial reactant ratio. The unreacted linker sites can subsequently participate in further polymerization. Extreme examples are the 1:5 (7000 arms and 35000 linkers) and 1:7 (7000 arms and 49000 linkers) cases. Here there is a large surplus of linker and one can see from Figure S5 and Table 2 that there are nearly 7000 bonds 


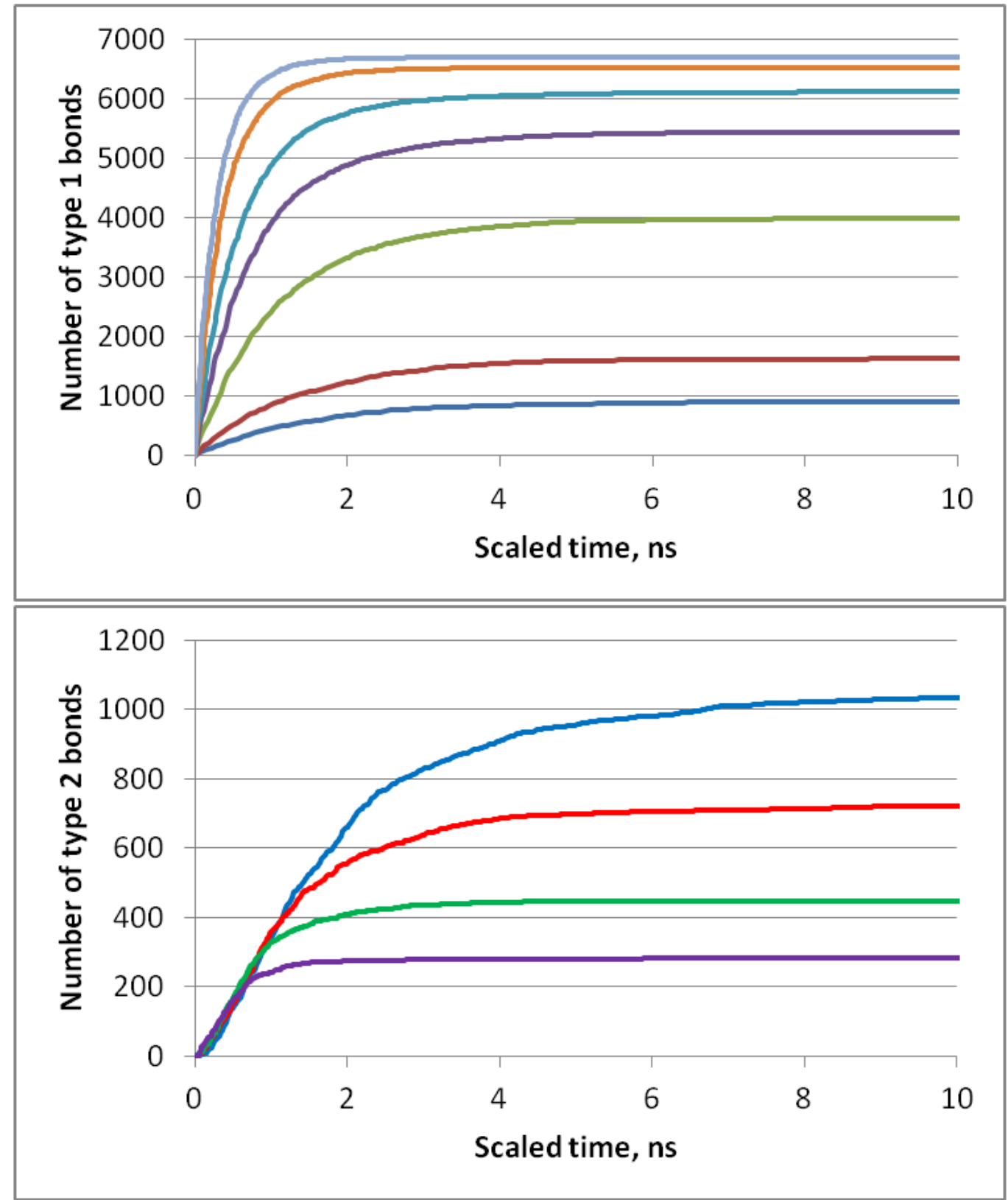

Figure S5: Number of bonds formed of type 1 (upper) and type 2 (lower) as a function of scaled time during simulations at intermediate total reactant density for a range of arm to linker reactant ratios. 
of type 1 (6540 for the 1:5 simulation and 6715 for the 1:7 simulation), and a much smaller number of bonds of type 2 (447 for the 1:5 and 284 for the 1:7 simulation), indicating that nearly every one of the 7000 arm molecules reacted with its own linker molecule to produce a nascent polymer with one arm and one linker. Note from Figure S5 Table 2 that as the relative amount of linker increases beyond an arm to linker ratio of 1:1, the number of bonds of type 2 actually decreases since it becomes less and less likely for an arm to combine with a linker that has already started to participate in a polymer. The resulting approximately 7000 reactive polymers with one linker and one arm will react further combining with remaining unreacted linker to produce, on average, polymers with 5 linkers per arm in the 1:5 case, and 7 linkers per arm in the 1:7 case.

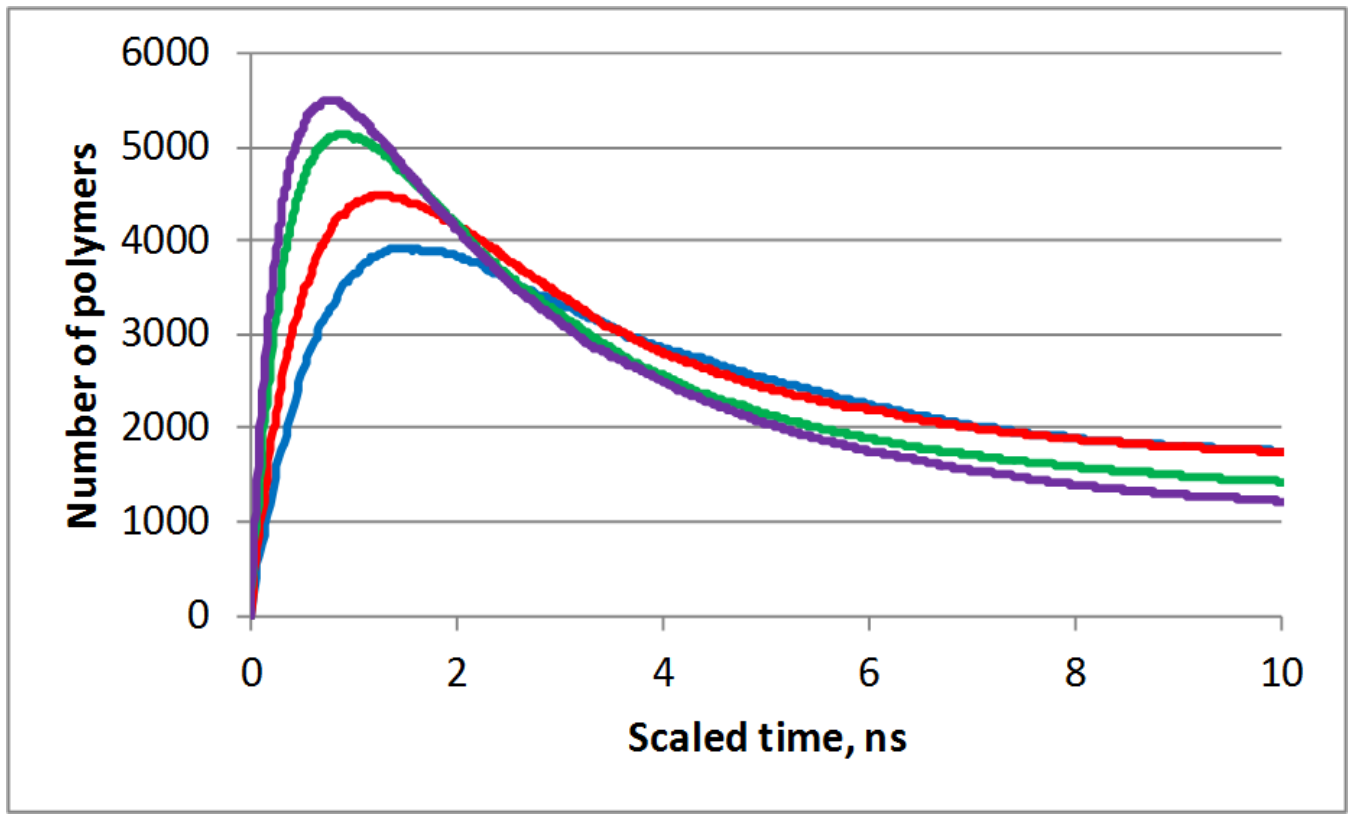

Figure S6: Number of polymers formed as a function of scaled time during simulations at intermediate reactant density for a range of arm to linker ratios.

Figure S6 shows the number of polymers (with unreacted molecules omitted) formed as a function of scaled time at intermediate reactant density for a range of arm to linker reactant ratios (from bottom to top at 1ns: 1:2, 1:3, 1:5 and 1:7). The number of polymers observed should scale with the 
number of initiators (arms), which is the same in all of these cases, so the curves can be compared directly. One can see that the number of polymers eventually approaches some value between 1000 and 2000 even though the amount of linker changes by a factor of 3.5 across these simulations. There is a systematic trend in the maxima of these curves that would approach 7000 in the limit of a large amount of linker, due to the fact that in this limit, each arm will initiate a new polymer rather than join an existing one. It is important to note that the formation of huge polymers that span the simulation cell was not observed at these intermediate reactant densities for any of these concentration ratios, unlike what was observed at higher reactant densities when the arm to linker ratio was 1:5 and where the number of polymer molecules dropped to very small numbers.

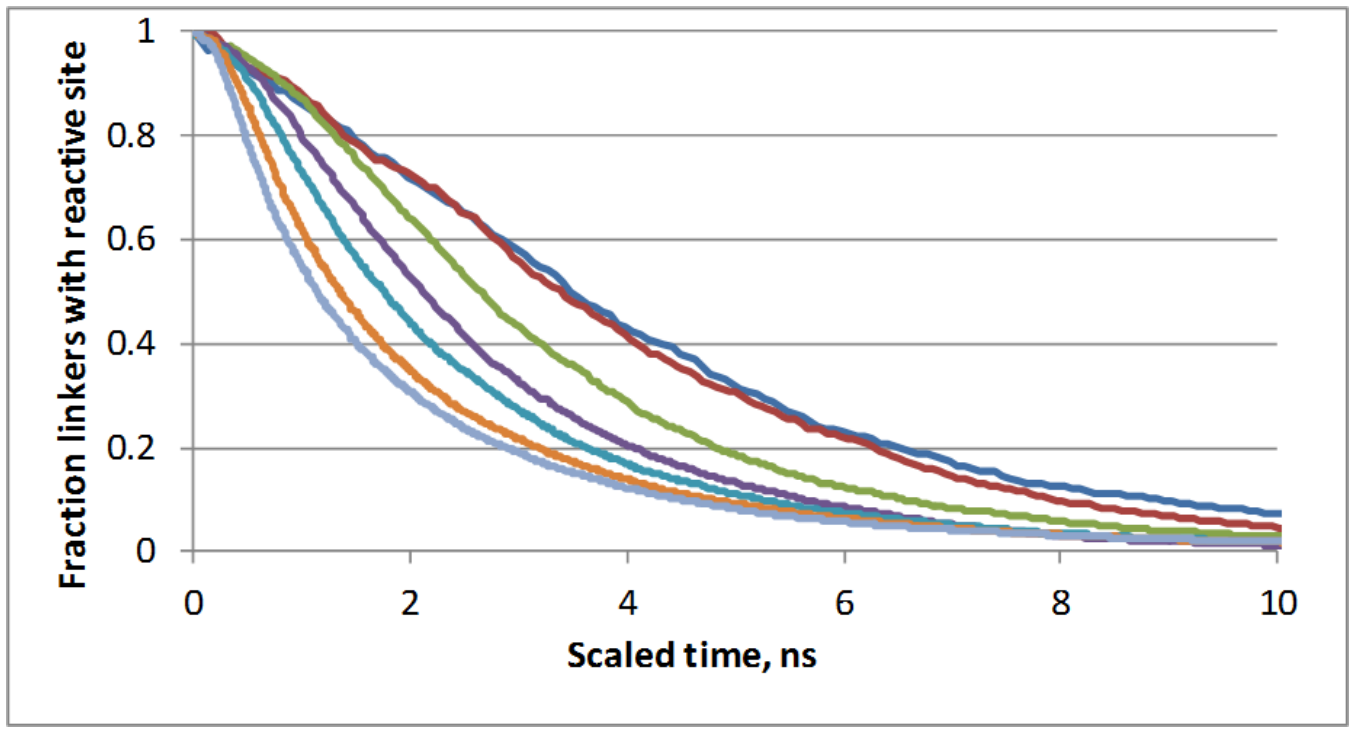

Figure S7: Fraction of the linker molecules that are participating in growing polymers and have one available unreacted carbonyl site.

Figure S7 shows the fraction of the linker molecules that are participating in growing polymers and have one available unreacted carbonyl site, as a function of scaled time at intermediate reactant density and various arm to linker reactant ratios, from top to bottom: 7:1, 7:2, 1:1, 1:2, 1:3, 1:5 and 1:7. (This data is similar to what is shown in the manuscript in Figure 21, but normalized differently.) At early times, each participating linker is joined by only one bond to its polymer, and this fraction is close to unity. Each linker 
that is added to these polymers from solution (bonds of type 3) moves this fraction closer to unity. But this fraction drops as arms are added to growing polymers (bonds of type 2), or intramolecular (type 4) or intermolecular (type 5) bonds form, because these processes consume unreacted carbonyl linker sites within a polymer molecule. The trend seen is best explained as a faster rate of formation for bonds of type 3 with increasing linker content.

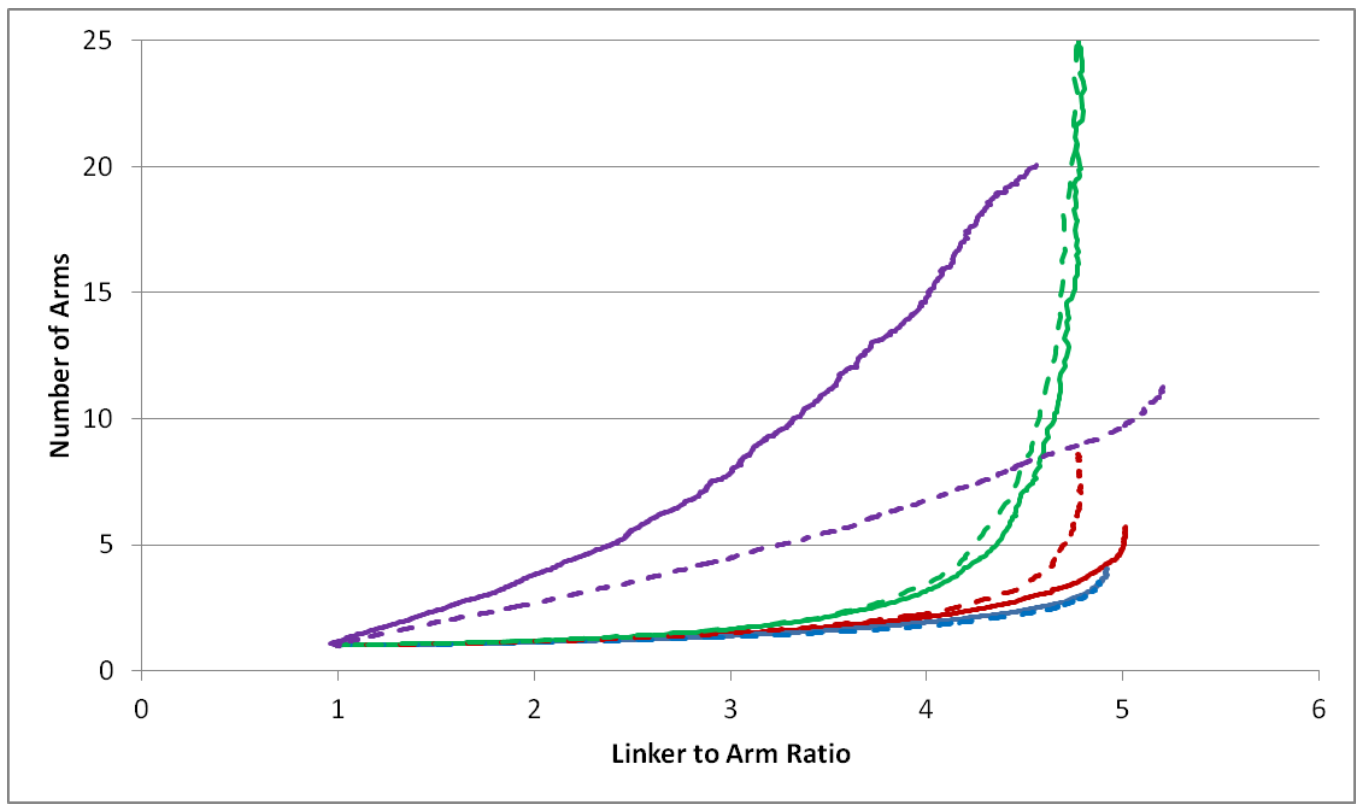

Figure S8: The evolution of the simulations projected onto a graph showing the polymer size, expressed as the average number of arms, and the composition, expressed as the number of linker components per arm.

Figure S8 shows the evolution of the simulations projected onto a graph showing the polymer size, expressed as the average number of arms, and the composition, expressed as the number of linker components per arm, during simulations where arm to linker reactant ratio was 1:5 at low (blue), medium (red) and higher reactant density (green) for short (solid) and longer (dashed) arms. Also shown (purple) is the evolution of two titration simulations performed with short (solid) and long (dashed) arm reactants. Averages of the arm content and linker to arm component ratios are number averages over all polymers observed at each time during the simulation. Early stages in the polymer growth corresponds to addition of linker to arms (average number 
of arms is constant, curves propagate to the right), and later to the aggregation of smaller polymers to make larger ones (composition is constant, curves propagate upward). Titration allows these processes to occur simultaneously, rather than sequentially, with a slope that depends on the density.

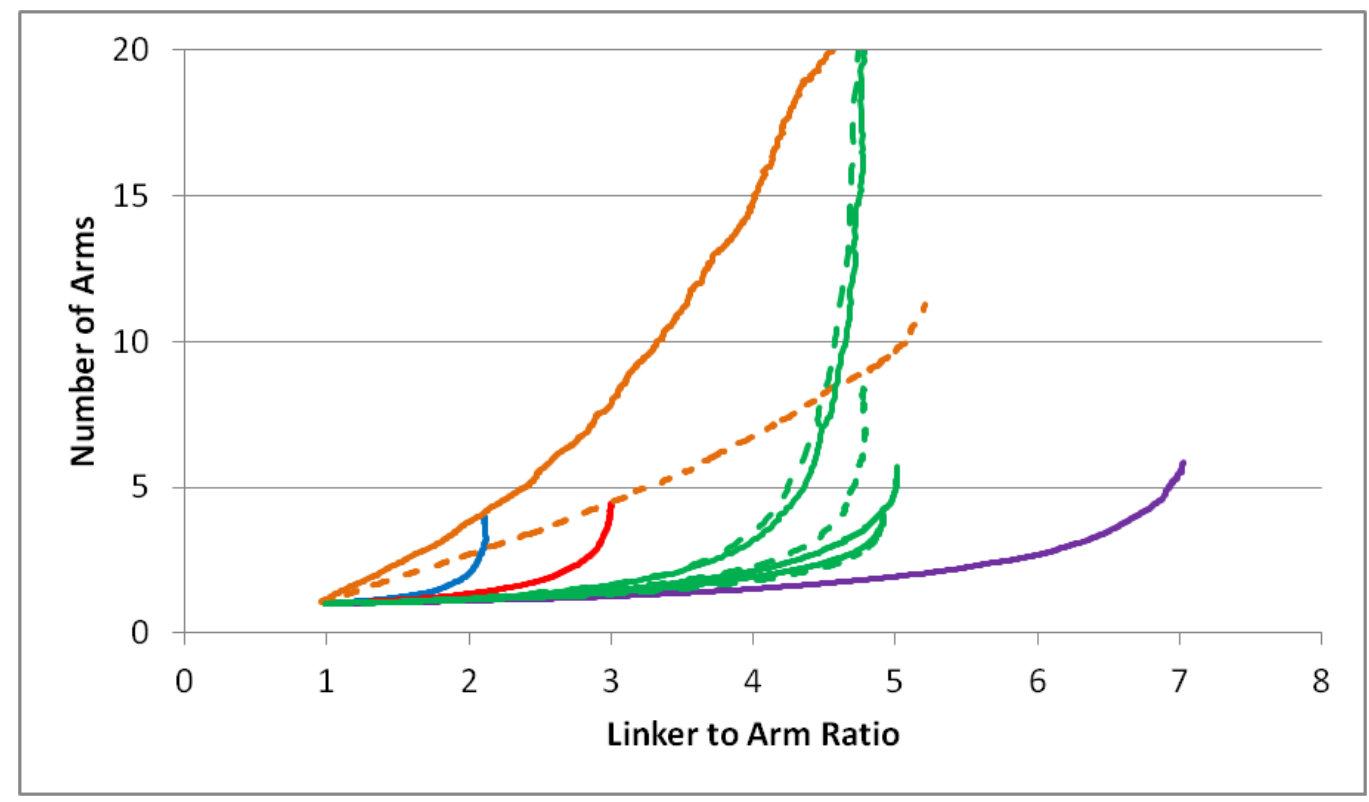

Figure S9: The evolution of the simulations projected onto a graph showing the polymer size, expressed as the average number of arms, and the composition, expressed as the number of linker components per arm.

Figure S9 shows the evolution of the simulations projected onto a graph showing the polymer size, expressed as the average number of arms, and the composition, expressed as the number of linker components per arm, during simulations where the arm to linker reactant ratio was varied from 1:2 (blue), 1:3 (red), 1:5 (green) and 1:7 (purple). The various green curves show the effect on arm count of changes in reactant concentration with lower concentration resulting in fewer arms. The orange lines represent the effect of gradual linker addition at two different reactant concentrations. Simulations with long arms are represented by dashed lines. Averages of the arm content and linker to arm component ratios are number averages over all polymers observed at each time during the simulation.

Figure S10 shows the evolution of the simulations projected onto a graph showing the polymer size, expressed as the average number of arms, and the 


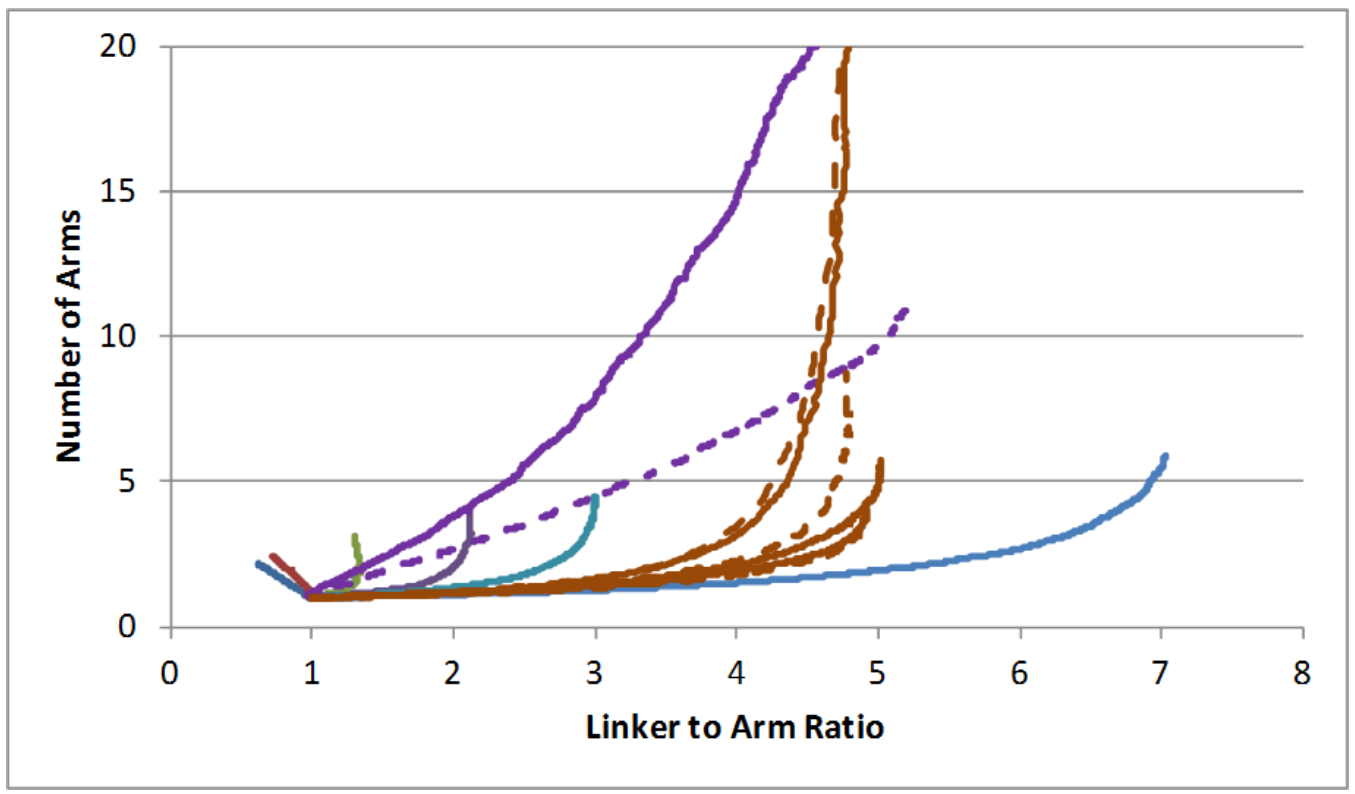

Figure S10: The evolution of the simulations projected onto a graph showing the polymer size, expressed as the average number of arms, and the composition, expressed as the number of linker components per arm.

composition, expressed as the number of linker components per arm, during simulations where the arm to linker reactant ratio was varied from 7:1 (far left, dark blue) to $7: 2,1: 1,1: 2,1: 3,1: 5$, and 1:7 (far right, blue) for short arms and intermediate reactant density. Six curves near the center in dark orange represent simulations with arm to linker content of 1:5 at different reactant densities. Also shown (purple, with larger numbers of arms) is the evolution of titration simulations where linker was added gradually during the simulation. Simulations with longer arms are indicated with dashed lines. Averages of the arm content and linker to arm component ratios are number averages over all polymers observed at each time during the simulation.

\section{Experimental Materials and Methods}

${ }^{1} \mathrm{H}$ NMR spectra were recorded on a Bruker Avance 2000 spectrometer operating at $400 \mathrm{MHz}$ (proton) and were referenced to internal solvent $\left(\mathrm{CDCl}_{3}\right.$, $\left.{ }^{1} \mathrm{H}=7.26 \mathrm{ppm}\right)$. All NMR spectra were recorded at room temperature using 
standard Bruker library pulse programs. All chemicals and solvents were purchased from Aldrich Chemical Co (Milwaukee, WI), unless stated otherwise. Deuteriated solvents were purchased from Cambridge Isotopes (Andover MA) and used as received. 4, 4'-Bicyclohexanone was supplied by TCI America and was used as received, $\alpha$-methoxy- $\omega$-hydroxy terminated poly(ethylene oxide) $(\mathrm{PEO}-\mathrm{OH})\left(\mathrm{M}_{\mathrm{n}} 5 \mathrm{kDa}, \mathrm{PDI}=1.02\right)$ was obtained from Fluka/SigmaAldrich, purified by azeotropic distillation from benzene and dried under vacuum prior to use. 1,5,7-Triazabicyclo[4.4.0]dec-5-ene (TBD) was purified by sublimation before use. Bis- $\epsilon$-caprolactone (BOD) was prepared from 4, $4^{\prime}$-bicyclohexanone according to earlier published procedure (vide infra). Anhydrous toluene, benzoic acid and diethyl ether were used as received.

Analytical gel permeation chromatography (GPC) was performed in THF using Waters high resolution columns HR1, HR2, HR4E and HR\%E (flow rates $1 \mathrm{~mL} / \mathrm{min}$ ) and peaks detected using either a Waters 996 diode array or a Waters 411 differential refractometer and calibrated polystyrene standards to determine molecular weights and polydispersities. Dynamic Light Scattering (DLS) measurements yielded values for $\mathrm{M}_{\mathrm{w}}, \mathrm{M}_{\mathrm{w}} / \mathrm{M}_{\mathrm{n}}$ and hydrodynamic radii $\left(R_{h}\right)$ using the described GPC column set with a Wyatt DAWN EOS multi-angle light scattering detector.

\section{Synthesis of Star Polymer with PEO arms}

Typical synthesis was performed as follows: in a glove box, to a solution of poly(ethylene oxide) arms ( $0.11 \mathrm{mmol}, 0.55 \mathrm{~g}$ ) (molecular weight $5 \mathrm{kDa})$ in $5.5 \mathrm{~g}$ of anhydrous dichloromethane (DCM) bis- $\epsilon$-caprolactone (BOD) $(0.84 \mathrm{mmol}, 0.19 \mathrm{~g})$ was added, followed by addition of $0.1 \mathrm{~g}$ of 5 wt\% solution of TBD in toluene. Reaction mixture was allowed to stir at $35 \mathrm{C}$, after 16 hours reaction was quenched with $20 \mathrm{mg}$ of benzoic acid and resulted solution was precipitated from cold diethyl ether. Obtained precipitate was filtered and dried at room temperature. The crude polymer was dissolved in $3 \mathrm{~mL}$ of DCM and $15 \mathrm{~mL}$ of diethyl ether was slowly added to a stirred solution. Resulted emulsion was allowed to settle for 2 hours, forming transparent oil at the bottom of the flask. The solution was decanted off and the oil was dissolved in a minimum amount of DCM, precipitated from ethyl ether, filtered and dried under vacuum for

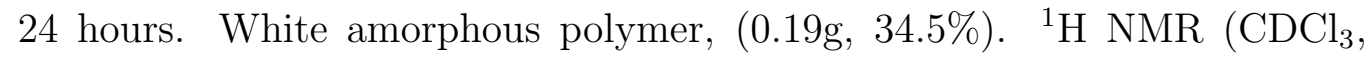
$400 \mathrm{MHz}): \quad \delta(\mathrm{ppm})=4.45-3.90\left(\mathrm{br}, 25 \mathrm{H},-\mathrm{CH}_{2}-\mathrm{CH}_{2}-\mathrm{OOC}-\right), 3.58$ (br, $386 \mathrm{H},-\mathrm{O}-\mathrm{CH}_{\mathbf{2}}-\mathrm{CH}_{\mathbf{2}}-\mathrm{O}-$, from the core $\left.-\mathrm{CH}_{\mathbf{2}}-\mathrm{OH}\right), 3.40$ (s, $3 \mathrm{H}, \mathrm{CH}_{3}-\mathrm{O}-\mathrm{CH}_{2}-$ ), 2.80-2.15 (br, $24 \mathrm{H},-\mathrm{CH}_{2}-\mathrm{CH}_{2}-\mathrm{COO}-$ ), 2.00-1.3 (br, $63 \mathrm{H},-\mathrm{OOC}-\mathrm{CH}_{2}-\mathrm{CH}_{2}-\mathrm{CH}-\mathrm{CH}_{2}-\mathrm{CH}_{2}-\mathrm{OOC}-$ ). All other star 
polymers were produced in a similar fashion. The ratio between the arm and the linker was adjusted accordingly.

The integrated NMR peaks suggest that the arms have $96(=384 / 4)$ $-\mathrm{CH} 2-\mathrm{CH} 2-\mathrm{O}-$ repeat units in the PEO arms. The $5 \mathrm{kDa}$ arms, however, are known to have about 114 such units. The discrepency is believed to be due to the broadening of the NMR signal due to protons that have hindered rotation, such as those close to the gelcore. The data also suggest an arm-to-linker ratio of about $6.3(=63 / 10)$, since there are ten protons in each linker contributing to the signal in the 2.00-1.3 chemical shift range. This is close to the 1:7.6 arm-to-linker ratio in the reaction mixture. However, NMR analysis of the other mixtures (1:2.5, 1:5, 1:10) also yields arm-to-linker ratios of $5.75,5.75$ and 6 , respectively, showing an unexpected lack of sensitivity to the initial arm-to-linker reactant mixture that might be explained by the fact that these measurements are averages over a distribution of many polymer sizes and that the broadening is different for large and small polymers.

Final product polymer molecular weights were estimated from the GPC elution times for the 1:2.5, 1:5, 1:7.5 and 1:10 arm-to-linker mixtures as $55.2,58.7,94.4$ and $118.0 \mathrm{kDa}$. These molecular weights should be viewed as approximate since the GPC calibration was based on linear polystyrene standards. However, with basic units consisting of one $5 \mathrm{kDa}$ PEO arm and its share of linker (MW=226 g/mole), these polymer masses imply arm counts of 10, 10, 14 and 16. Even if the basic unit in each case was taken to be an arm and six linkers, as suggested by the NMR, this implies arm counts of 9 , 9, 15 and 19. Subject to experimental uncertainty, these interpretations are consistent with each other and with simulation results, and show expected increase in polymer size with reactant concentration and linker content. 